\title{
Some like it cold: molecular emission and effective dust temperatures of dense cores in the Pipe Nebula ${ }^{\star}$
}

\author{
Jan Forbrich ${ }^{1,2}$, Karin Öberg ${ }^{2}$, Charles J. Lada ${ }^{2}$, Marco Lombardi $^{3}$, Alvaro Hacar ${ }^{1}$, João Alves ${ }^{1}$, and Jill M. Rathborne ${ }^{4}$ \\ ${ }^{1}$ University of Vienna, Department of Astrophysics, Türkenschanzstr. 17, 1180 Vienna, Austria \\ e-mail: jan. forbrich@univie.ac.at \\ 2 Harvard-Smithsonian Center for Astrophysics, 60 Garden Street, Cambridge MA 02138, USA \\ 3 University of Milan, Department of Physics, via Celoria 16, 20133 Milan, Italy \\ ${ }^{4}$ CSIRO Astronomy and Space Science, Epping, Sydney NSW 1710, Australia
}

Received 31 March 2014 / Accepted 29 May 2014

\section{ABSTRACT}

\begin{abstract}
Aims. The Pipe Nebula is characterized by a low star-formation rate and is therefore an ideal environment to explore how initial conditions, including core characteristics, affect star-formation efficiencies.

Methods. In a continued study of the molecular core population of the Pipe Nebula, we present a molecular-line survey of 52 cores. Previous research has shown a variety of different chemical evolutionary stages among the cores. Using the Mopra Radio Telescope, we observed the ground rotational transitions of $\mathrm{HCO}^{+}, \mathrm{H}^{13} \mathrm{CO}^{+}, \mathrm{HCN}, \mathrm{H}^{13} \mathrm{CN}, \mathrm{HNC}$, and $\mathrm{N}_{2} \mathrm{H}^{+}$. These data are complemented with near-infrared extinction maps to constrain the column densities, effective dust temperatures derived from Herschel data, and $\mathrm{NH}_{3}{ }^{-}$ based gas kinetic temperatures.

Results. The target cores are located across the nebula, span visual extinctions between 5 and 67 mag, and effective dust temperatures (averaged along the lines of sight) between 13 and $19 \mathrm{~K}$. The extinction-normalized integrated line intensities, a proxy for the abundance in constant excitation conditions of optically thin lines, vary within an order of magnitude for a given molecule. The effective dust temperatures and gas kinetic temperatures are correlated, but the effective dust temperatures are consistently higher than the gas kinetic temperatures. Combining the molecular line and temperature data, we find that $\mathrm{N}_{2} \mathrm{H}^{+}$is only detected toward the coldest and densest cores, while other lines show no correlation with these core properties.

Conclusions. Within this large sample, $\mathrm{N}_{2} \mathrm{H}^{+}$is the only species to exclusively trace the coldest and densest cores, in agreement with chemical considerations. In contrast, the common high-density tracers $\mathrm{HCN}$ and $\mathrm{HNC}$ are present in a majority of the cores, demonstrating the utility of these molecules for characterizing cores over a wide range of extinctions. The correlation between the effective dust temperatures and the gas kinetic temperatures suggests that the former are dominated by dust that is both dense and thermodynamically coupled to the dense gas traced by $\mathrm{NH}_{3}$. A direct use of the effective dust temperatures in a determination of dust column densities from dust emission measurements would, however, result in an underestimate of the dust column densities.
\end{abstract}

Key words. stars: formation - dust, extinction - radio lines: ISM - submillimeter: ISM

\section{Introduction}

The Pipe Nebula with its population of dense low-mass cores is an ideal environment to study the initial conditions of star formation. The extinction maps of Lombardi et al. (2006) and Román-Zúñiga et al. (2010) have shown that the Pipe Nebula as a whole is similar to the $\rho$ Oph star-forming region in mass, size and distance $(d=130 \mathrm{pc})$, and yet it has almost no active star formation (Forbrich et al. 2009, 2010). Using extinction mapping, $\mathrm{C}^{18} \mathrm{O}$, and $\mathrm{NH}_{3}$ observations in particular, the core population has been characterized in detail. Muench et al. (2007) confirmed the nature of the core population as consisting of distinct cores and not chance superpositions in the interstellar medium. In a study of the core mass function, as derived from extinction mapping, Alves et al. (2007) found that the core mass function is similar in shape to the stellar initial mass function, from which it is different only by a scaling factor in mass. Lada et al. (2008) found that most of the cores are supported by thermal pressure. Additionally, Rathborne et al. (2008) presented a survey of $\mathrm{NH}_{3}, \mathrm{CCS}$, and $\mathrm{HC}_{5} \mathrm{~N}$ emission toward some of the cores, and Rathborne et al. (2009) obtained an improved census of the

* Table 1 is available in electronic form at http://www . aanda.org core population. They concluded that there are 134 physically distinct dense cores associated with the Pipe Nebula. They also confirmed the similarity in shape of the core mass function and the stellar initial mass function.

The large number and diverse set of well-characterized cores makes the Pipe Nebula a great laboratory to probe the connection between physical and chemical conditions in cores. Observationally, this can be done using a combination of dust extinction and emission maps, as well as molecular lines that trace dense molecular gas. Commonly used dense gas tracers can be divided into three categories: 1. rare isotopologs of molecules with low dipole moments that are generally distributed and excited; 2 . molecules with high dipole moments that are generally distributed, but only excited at high densities; and 3. molecules that are only observable in high-density cores because of chemical considerations.

Rare isotopologs of $\mathrm{CO}$ are the most common probes that fall into the first category. These molecules will form at very low extinctions and have low critical densities, but because the lines can remain optically thin in high column density lines of sight, the emission will trace higher column density material and may thus be good to simultaneously study cloud material 
and identify cores that span a wide range of densities. HCN, $\mathrm{HNC}, \mathrm{HCO}^{+}$and their isotopologs have higher critical densities, and their emission is expected to pinpoint higher density gas rather than diffuse emission from the surrounding lower density cloud. They are therefore probably useful to characterize core material at a range of core densities above a certain threshold $\left(\sim 10^{4}-10^{7} \mathrm{~cm}^{-3}\right.$, depending on the excitation conditions of the molecule and transition). Finally, $\mathrm{N}_{2} \mathrm{H}^{+}, \mathrm{NH}_{3}$, and deuterated molecules are only expected in the coldest and densest cores because they rely on $\mathrm{CO}$ freeze-out to reach observable abundances. This therefore makes them unique tracers of the coldest and densest cores (Bergin et al. 2001, 2002; Tafalla et al. 2006). The goal of this study is to explore the molecular emission of a subset of these molecules toward a large sample of cores in a single star-forming region, the Pipe Nebula, to test the current ideas on the utility of these molecules as different kinds of core probes.

The molecular content in a subset of these cores has been explored before. Studying the evolutionary stages of various cores in the Pipe Nebula, Frau et al. (2010) presented molecular-line observations of four of these cores. Based on observations of 11 transitions of various molecules and a chemical-clock analysis, the authors concluded that the cores are all young, but in different evolutionary stages. Subsequently, Frau et al. (2012a) extended this survey to a total of 14 sources and multiple transition lines. They found a rich chemistry and divided the sources into three groups - diffuse, oxo-sulfurated, and deuterated cores. The first group shows only few emission lines in the most abundant species. The second group, at a higher column density, shows emission from oxo-sulfurated molecules, while the third group, at the highest column densities, additionally shows emission from deuterated species. In a third paper, Frau et al. (2012b) extended the detailed molecular line survey of the first paper from four to a total of nine sources, which again fall into three groups. The total sample of cores considered in these papers consists of 15 cores, as marked in Table 1.

In this paper, we study chemical differences across the core population of the Pipe Nebula. We observed six molecular line transitions toward 52 out of 134 Pipe cores. In addition, we use near-infrared extinction maps and submillimeter data from the Herschel satellite to derive effective dust temperatures.

\section{Observations}

\subsection{Mopra millimeter-line data}

Observations were obtained in 2007 July 3-8 and 2008 July 2025 at the 22-m Mopra Radio Telescope. They consist of positionswitched single pointings of individual cloud cores in the Pipe Nebula with positions identified from extinction maps (Alves et al. 2007; Rathborne et al. 2009) as listed in Table 1. The $8 \mathrm{GHz}$ MOPS spectrometer was used to simultaneously observe the ground transitions of $\mathrm{HCN}, \mathrm{H}^{13} \mathrm{CN}, \mathrm{HCO}^{+}, \mathrm{H}^{13} \mathrm{CO}^{+}, \mathrm{HNC}$, and $\mathrm{N}_{2} \mathrm{H}^{+}$, covering a frequency range of about $86-93 \mathrm{GHz}$. Each of these lines was covered with one spectral window, $137.5 \mathrm{MHz}$ wide with 4096 channels. This produced a velocity resolution of about $0.11 \mathrm{~km} \mathrm{~s}^{-1}$. The full width at half-maximum (FWHM) Mopra beam size is $\sim 36^{\prime \prime}$ (Ladd et al. 2005) at a frequency of $86 \mathrm{GHz}$, corresponding to an absolute scale of $0.02 \mathrm{pc}$ at the distance of the Pipe Nebula (130 pc, Lombardi et al. 2006). The telescope pointing was checked approximately every hour using a suitably bright, nearby maser.

The spectra were reduced using the ATNF Spectral Analysis Package (ASAP). All spectra are in $T_{\mathrm{A}}^{*}$ with a median noise of $0.05 \mathrm{~K}$ per channel. We have searched all spectra for detections at about the system velocity and with more than one channel above the $3 \sigma$ threshold. Integrated intensities (in $T_{\mathrm{A}}^{*}$ ) were obtained from the spectra, including hyperfine components where applicable. Corresponding error bars were conservatively calculated over the full line widths using error propagation, assuming an error for every spectral bin that is equivalent to the rms noise of the spectrum. The numerical values of errors and upper limits vary because of the differences in integration time and weather conditions.

\subsection{Herschel submillimeter continuum data}

In addition to the Mopra observations we used an effective dust temperature map that was generated from Herschel multiband submillimeter observations, as calibrated with Planck and extinction data. These effective dust temperatures are based on fits of the spectral energy distribution with a modified blackbody and represent weighted averages of the dust temperatures along the lines of sight, where warmer dust is weighted more than colder dust because the emission is stronger. The procedure is explained in detail in Lombardi et al. (2014). Briefly, the optical depth, temperature, and spectral index maps from the Planck mission are used to predict and correct the Herschel fluxes in the various bands to obtain absolute calibration information. Optical depth and effective dust temperature maps are then obtained in graybody fits with these two parameters while assuming a local value for the dust spectral index $\beta$ as taken from Planck/IRAS maps. Finally, the submillimeter opacity is tied to a near-infrared extinction map in a linear fit to obtain a column density map from the Herschel data. The angular resolution of the resulting column density and temperature maps is 36" FWHM. While we can derive nominal temperature uncertainties between $0.06 \mathrm{~K}$ and $0.40 \mathrm{~K}$, as listed in Table 1 , the total error budget is most likely dominated by systematics. These include temperature or $\beta$ gradients along the line of sight, local spatial changes of $\beta$, other changes in the extinction law, or inaccuracies in the absolute flux calibration of Herschel. While these are hard to quantify, we point out that we are mainly using relative temperature measurements here, which minimizes the effect of these systematics.

\section{Results and discussion}

\subsection{Line intensities across the Pipe Nebula core population}

Integrated line intensities depend on a combination of total column density, molecular abundances, and excitation conditions (typically dominated by temperature and density). As a proxy for molecular abundances we have divided the line intensities by the visual extinction in each line of sight (similar to Frau et al. 2012a), providing extinction-normalized line intensities that for optically thin lines should only depend on excitation and chemistry. For each core the integrated intensities are divided by the value of the corresponding pixel in the extinction maps of Román-Zúñiga et al. (2010), or, in two cases ${ }^{1}$, Lombardi et al. (2006) with its wider coverage. These extinction maps have an angular resolution of $20^{\prime \prime}$ and $60^{\prime \prime}$ FWHM, respectively.

The two extinction values separating the three groups of cores in the Frau et al. papers are $A_{V} \sim 22.5 \mathrm{mag}$ and $A_{V} \sim$ 15.0 mag. Applying these numbers to our sample, we have four sources in the highest-extinction group, six sources in the second group, and 43 sources in the lowest-extinction group. Note that all 15 sources in the Frau et al. papers also appear in our sample.

Marked in Table 1. 
J. Forbrich et al.: Some like it cold

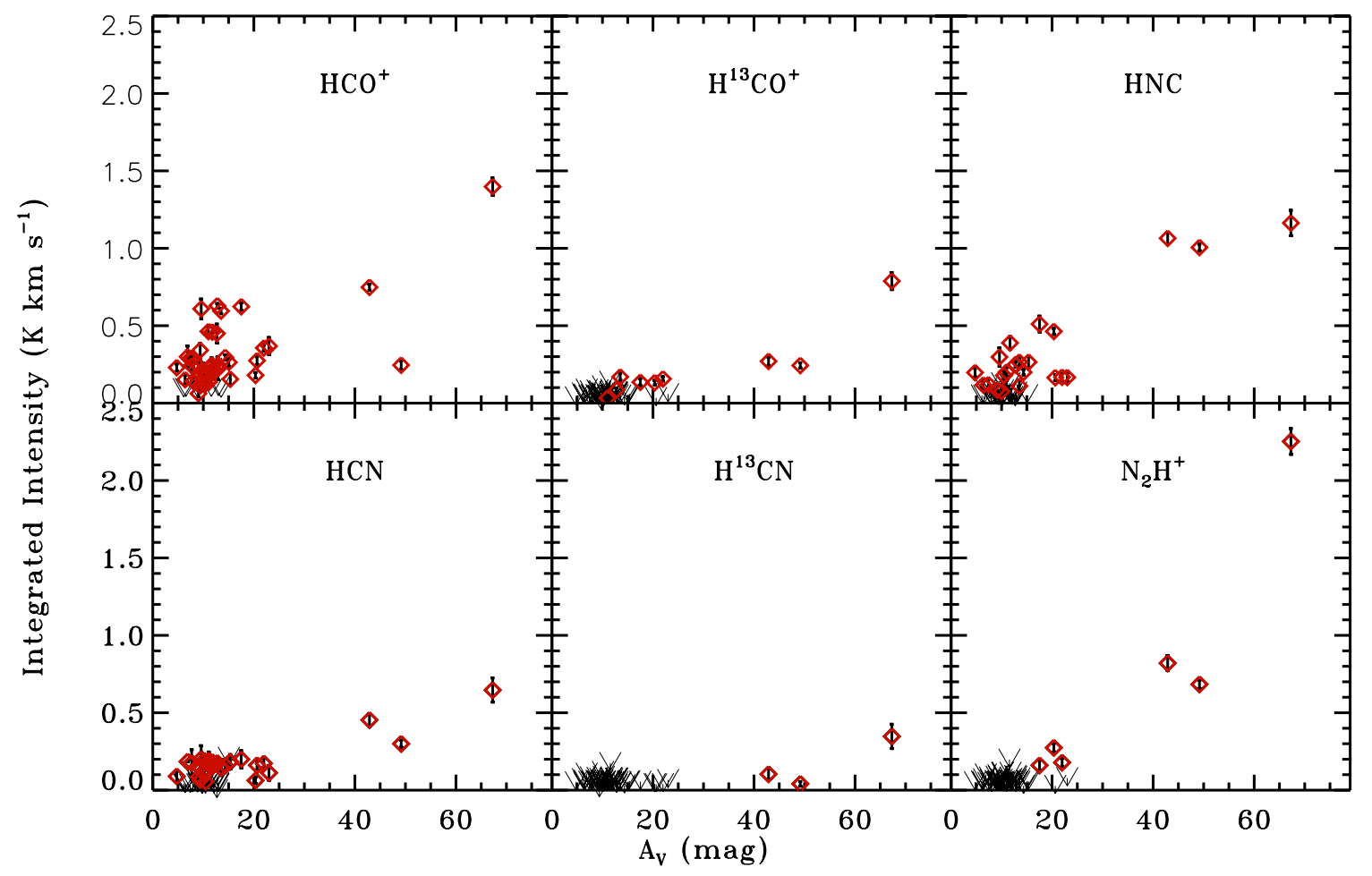

Fig. 1. Correlation of $\int T_{\mathrm{A}}^{*}$ of each transition with $A_{V}$, including upper limits.

The two most frequently detected lines in our sample, above $3 \sigma$, are $\mathrm{HCO}^{+}$and $\mathrm{HCN}$ with 43 and 29 detections, respectively. In their isotopologs $\mathrm{H}^{13} \mathrm{CO}^{+}$and $\mathrm{H}^{13} \mathrm{CN}$, there are only 9 and 3 detections, respectively. Finally, we detect HNC toward 22 and $\mathrm{N}_{2} \mathrm{H}^{+}$toward six cores.

Our results are listed in Table 1, and we show a plot of integrated line intensities over extinction in Fig. 1. The three cores with the highest extinction levels $\left(A_{V}>40 \mathrm{mag}\right)$, namely Barnard 59, the Pipe molecular ring, and FeSt 1-457, are detected in all lines, while many of the less extincted cores have upper limits in some or all of the transitions. HCN, HNC, and $\mathrm{HCO}^{+}$are detected across the full range of extinctions in our sample, with several non-detections at low extinction levels $\left(A_{V}<15 \mathrm{mag}\right) . \mathrm{H}^{13} \mathrm{CN}$ and to a lesser degree $\mathrm{H}^{13} \mathrm{CO}^{+}$are only detected toward the highest extinctions. $\mathrm{N}_{2} \mathrm{H}^{+}$is detected only toward cores with high visual extinction of $A_{V}>17 \mathrm{mag}$. Two of the high-extinction cores do not display $\mathrm{N}_{2} \mathrm{H}^{+}$emission, however, and we discuss these below after we introduce the core temperatures.

The extinction-normalized integrated line intensities as a function of extinction are shown in Fig. 2. $\mathrm{HCO}^{+}$and $\mathrm{HCN}$ show a large scatter at low extinctions $\left(A_{V}<20 \mathrm{mag}\right)$ around means that are higher than for the high extinction cores, meaning that there is a marginal decrease. Some of the scatter is due to noise, but some may also be due to abundance variations and different excitation conditions in the lower density cores. The decreasing normalized line intensities with extinction might be due to a decreasing abundance, but here these are instead indicative of lines that are becoming increasingly optically thick, thus no longer tracing the total column. Indeed, the intensity ratios of the isotopologues are far lower than what would be expected for optically thin transitions of $\mathrm{HCO}^{+}$and $\mathrm{HCN}$. This result is supported by the flat trends of $\mathrm{H}^{13} \mathrm{CO}^{+}$and $\mathrm{H}^{13} \mathrm{CN}$ with extinction, which is expected for optically thin lines that are present at a constant abundance across the full range of core properties. While for $\mathrm{HCO}^{+}$the decrease of the extinction-normalized integrated intensities might partly also be due to depletion, it is therefore more likely due to an optical depth effect since the decrease is not confirmed by the limited sample of $\mathrm{H}^{13} \mathrm{CO}^{+}$detections. However, deeper observations of $\mathrm{H}^{13} \mathrm{CO}^{+}$at the lowermost extinctions would be required for a definitive test.

$\mathrm{N}_{2} \mathrm{H}^{+}$is the only molecule whose normalized emission clearly increases with extinction. For visual extinctions above $A_{V}=15 \mathrm{mag}$, increasing abundances for $\mathrm{N}_{2} \mathrm{H}^{+}$with extinction were already pointed out by Bergin et al. (2001). Since the $\mathrm{N}_{2} \mathrm{H}^{+}$critical density is similar to those of $\mathrm{HCN}$ and $\mathrm{HNC}$ this may have a chemical rather than an excitation origin, particularly when considering that the excitation temperature of $\mathrm{N}_{2} \mathrm{H}^{+}$ in dense cores has been found to be fairly constant (Caselli et al. 2002).

\subsection{Core properties as a function of temperature}

The effective dust temperatures from Herschel allow us to better understand the chemistry of these cores. Individual temperatures were extracted for each core at its observed position. It becomes apparent immediately that there is a strong anti-correlation between the core column density, as traced by the extinction map, and the effective dust temperature (Fig. 3). This indicates that the cores with the highest levels of extinction do contain the coldest material. The high column densities are thus not a consequence of a long column of warm diffuse emission; instead, the high column densities correspond to spatially concentrated high volume densities, shielding the inner regions of the cores from heating by external radiation. Note that unlike previous studies of individual cores (e.g., Ward-Thompson et al. 2002; Gonçalves et al. 2004), the data points shown in Fig. 3 represent distinct cores and not a single coherent region. As a result, a direct comparison may not be particularly meaningful.

Since the effective dust temperatures may be biased by the line-of-sight averaging discussed above, we compared them with 
A\&A 568, A27 (2014)

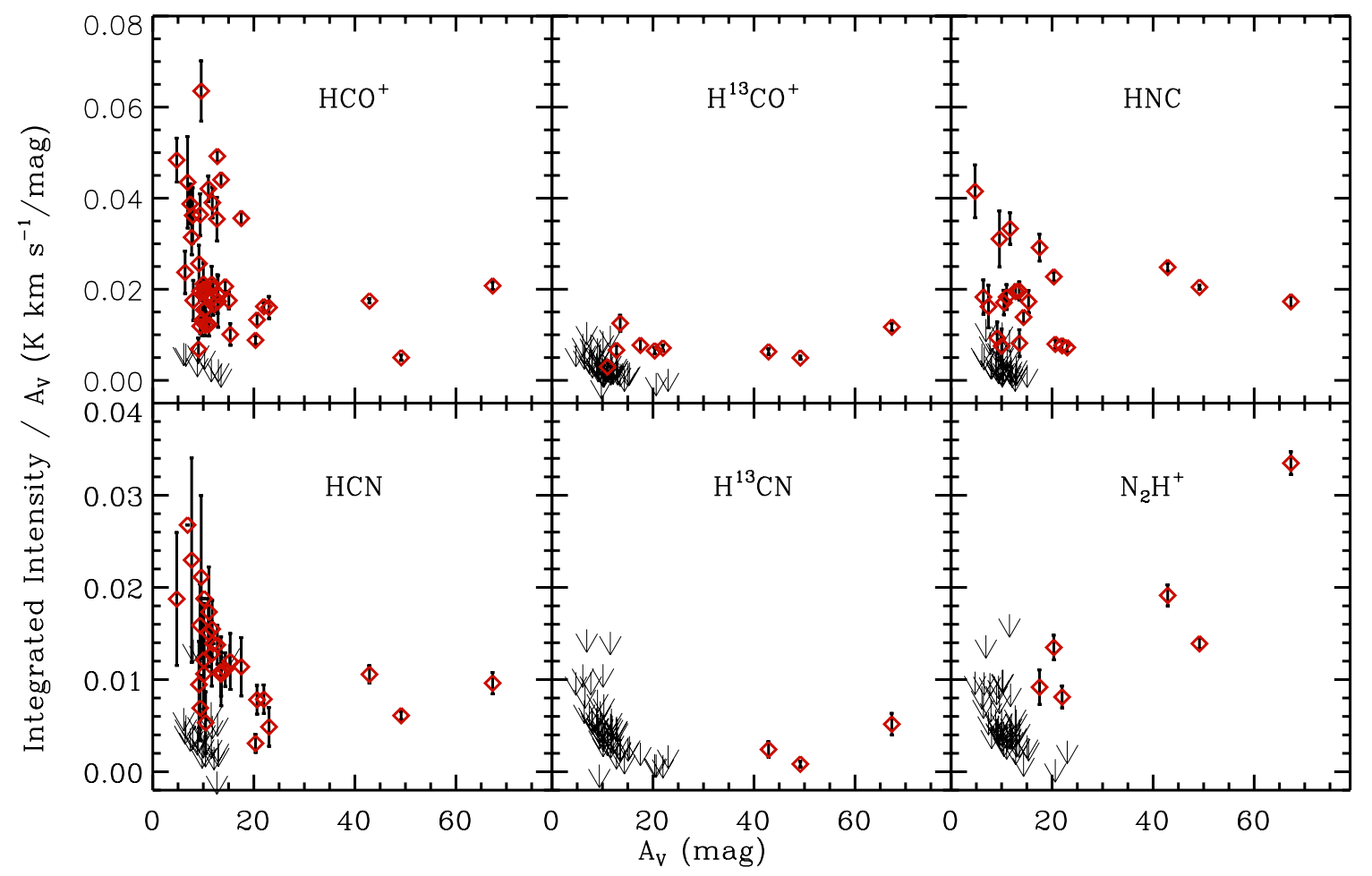

Fig. 2. Correlation of $\int T_{\mathrm{A}}^{*} / A_{V}$ of each transition with $A_{V}$, including upper limits.

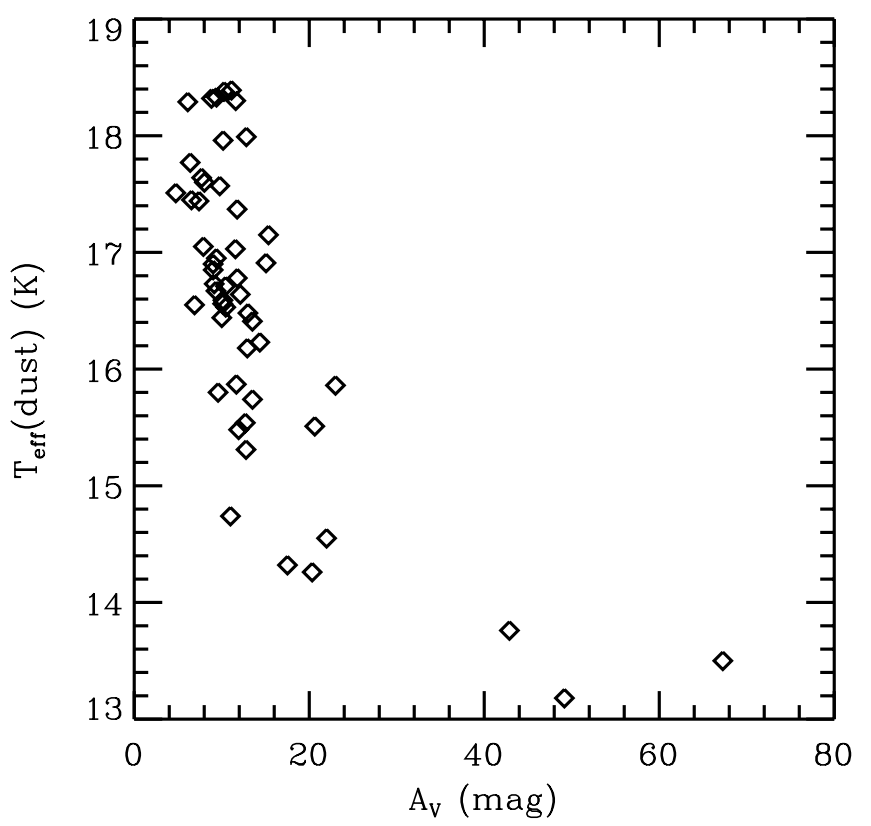

Fig. 3. Correlation of the effective dust temperature $T(\mathrm{~K})$ and the core visual extinction $A_{V}$ (mag).

gas kinetic temperatures derived from dense-gas-tracing $\mathrm{NH}_{3}$ observations. Assuming thermodynamic coupling of dense dust and gas, the two quantities should be correlated, and if the dust emission is dominated by the core, the two temperatures should be almost identical. Rathborne et al. (2008) present measurements of the $\mathrm{NH}_{3}$ gas temperatures for some of the Pipe cores, and in Fig. 4, we show a direct comparison of the gas kinetic temperature $T_{\text {gas }}$ from Rathborne et al. (2008) and the corresponding effective dust temperature. Figure 4 shows that while

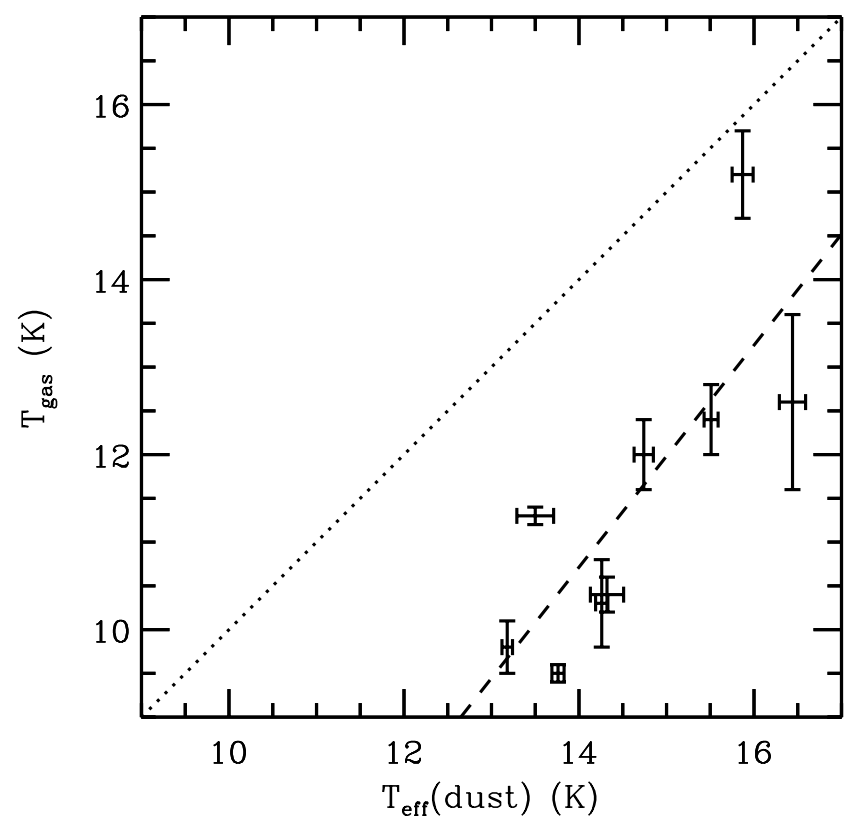

Fig. 4. Correlation of $T_{\mathrm{K}}$ from $\mathrm{NH}_{3}$ measurements reported by Rathborne et al. (2008) and the effective dust temperatures derived from Herschel data. The gas temperatures from $\mathrm{NH}_{3}$ are always lower than the effective dust temperatures. The dashed line shows a linear fit, and the dotted line indicates a direct correspondence of the two temperatures.

there is a direct linear correlation between the two quantities (dashed line), the effective dust temperatures are consistently higher than the gas temperatures, as expected from line-of-sight averaging of the dust that not only traces the densest and coldest parts of the clouds where dust and gas temperatures presumably are more closely coupled. Additionally, the dynamic range in the 


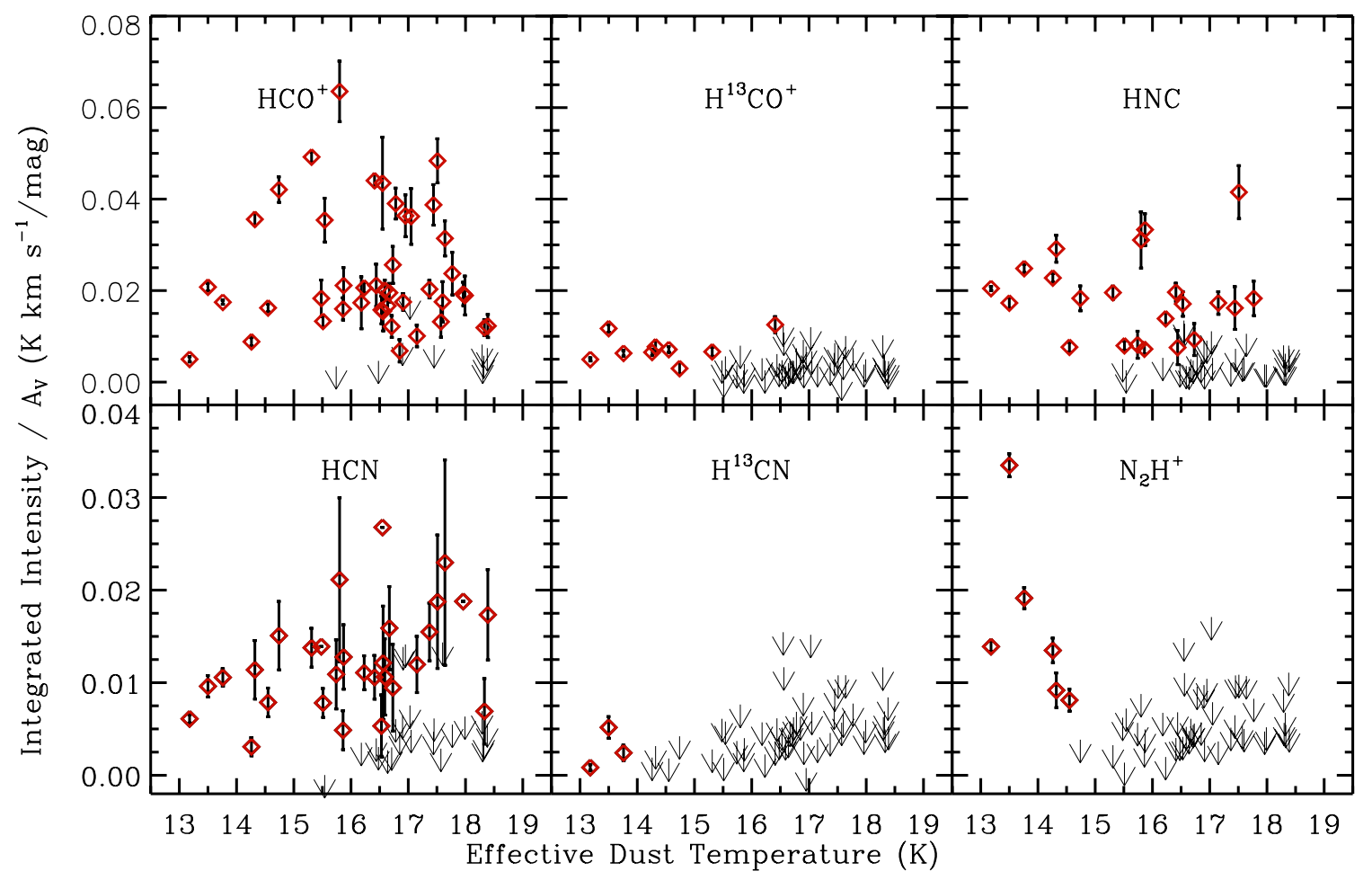

Fig. 5. Correlation of $\int T_{\mathrm{A}}^{*} / A_{V}$ of each transition with temperature, including upper limits.

$\mathrm{NH}_{3}$ temperatures is almost twice that of the effective dust temperatures, although that difference is mostly due to a single data point that also has the largest offset from the linear fit.

Nevertheless, the linear relation between the two sets of temperature measurements underlines that the effective dust temperatures are physically meaningful for our core sample and dominated by dust that is thermodynamically coupled to the dense gas. Such coupling is expected for volume densities above $10^{5 \ldots 6} \mathrm{~cm}^{-3}$ (Burke \& Hollenbach 1983; Goldsmith 2001). While these densities are higher than those derived from the $\mathrm{NH}_{3}$ observations, which are on the order of $10^{4} \mathrm{~cm}^{-3}$ (Rathborne et al. 2008), it seems plausible that higher densities might be reached in the interior of these cores. Thus, while the meaning of an absolute effective dust temperature is not obvious because of the line-of-sight averaging, the use of effective dust temperatures for relative comparisons within the core sample presented here appears to be justified. A direct use of the effective dust temperatures in a derivation of the dust column densities from dust emission measurements would, however, result in an underestimate of the dust column densities.

In Fig. 1, we show the integrated intensities in all six transitions as a function of column density, as traced by the extinction maps in $A_{V}$ (mag). All integrated line intensities increase with extinction, but the rate of increase differs by molecular transition. Additionally, in Fig. 5, we plot the extinction-normalized integrated line intensities as a function of the effective dust temperatures of the cores. The effective dust temperatures range from about $13 \mathrm{~K}$ to $19 \mathrm{~K}$. Interestingly, the coldest cores are detected in all six transitions, while that is not the case for the warmer cores. The trend is particularly clear in $\mathrm{N}_{2} \mathrm{H}^{+}$where only the coldest cores of the sample have been detected. They have temperatures between $13.2 \mathrm{~K}$ and $14.6 \mathrm{~K}$, which is colder than the temperatures of the above-mentioned undetected highextinction cores $(15.5 \mathrm{~K}$ and $15.9 \mathrm{~K})$, none of which are known to contain embedded sources (Forbrich et al. 2009). There is a tentative trend in $\mathrm{HCO}^{+}$and $\mathrm{HCN}$ to lower extinctionnormalized emission with lower temperatures. If the lines are optically thick, as indicated in some cases (see above), then the emission would mainly trace the temperature, explaining this correlation. The detections in $\mathrm{HCO}^{+}, \mathrm{HCN}$, and $\mathrm{HNC}$ span the full temperature range. While $\mathrm{H}^{13} \mathrm{CO}^{+}$and $\mathrm{H}^{13} \mathrm{CN}$ are preferentially detected at lower temperatures, this is mostly a column density effect since colder cores have higher column densities (Fig. 3).

Our finding that only the coldest cores are detected in $\mathrm{N}_{2} \mathrm{H}^{+}$ corroborates a recent similar indication of a correlation between the modeled central temperature of Taurus cores and the presence of $\mathrm{N}_{2} \mathrm{H}^{+}$emission: Marsh et al. (2014) found that the seven $\mathrm{N}_{2} \mathrm{H}^{+}$detections in their sample (from Hacar et al. 2013) include four out of the five coldest cores, and they note that $\mathrm{N}_{2} \mathrm{H}^{+}$ detections thus seem to be preferentially associated with the coldest cores. Here, we find a direct correlation with the more easily observable effective dust temperature that is not as modeldependent as the central core temperature; it is simply based on a modified blackbody fit to the submillimeter spectral energy distribution.

\section{Summary and conclusions}

We have observed 52 low-mass molecular cores in the Pipe Nebula in the ground rotational transitions of $\mathrm{HCN}, \mathrm{H}^{13} \mathrm{CN}$, $\mathrm{HCO}^{+}, \mathrm{H}^{13} \mathrm{CO}^{+}, \mathrm{HNC}$, and $\mathrm{N}_{2} \mathrm{H}^{+}$. These molecular-line observations were interpreted in the context of the dust column densities and effective dust temperatures of the cores, as derived from extinction maps and Herschel submillimeter mapping, respectively. The column densities and effective dust temperatures toward the cores are generally anti-correlated, that is, the cores with the highest levels of extinction also have the lowest dust temperatures. This is consistent with an interpretation that the 
highest-extinction cores indeed contain both cold and dense material. A comparison of effective dust temperature and $\mathrm{NH}_{3}$ gas kinetic temperatures for a subset of our sample also demonstrated that the two quantities are correlated, indicating that the effective dust temperature is dominated by dust that is thermodynamically coupled to dense gas, even if the effective dust temperature is elevated by the inclusion of hotter dust in the line-ofsight averaging. At least relative statements based on effective dust temperatures are thus physically meaningful.

While all integrated line intensities increase with line-ofsight extinction, the rate of increase is different for different molecules. $\mathrm{HCO}^{+}$and $\mathrm{HCN}$ emission, normalized with extinction, decreases with increasing extinction, most likely because the lines become optically thick. This is demonstrated by $\mathrm{H}^{13} \mathrm{CO}^{+}$and $\mathrm{H}^{13} \mathrm{CN}$, where the emission, normalized with extinction, is roughly constant within the sample, indicative of a constant abundance and optically thin lines. $\mathrm{N}_{2} \mathrm{H}^{+}$emission normalized with extinction increases with extinction, which is indicative of overproduction of this molecule relative to the others toward the densest cores. Based on previous observations and chemical modeling, $\mathrm{N}_{2} \mathrm{H}^{+}$will only become observable in dense regions when the temperature is low enough for $\mathrm{CO}$ to freeze out ( $T \sim 20 \mathrm{~K}$; Bisschop et al. 2006). Supporting this theory, $\mathrm{N}_{2} \mathrm{H}^{+}$ emission (absolute and normalized) is generally anti-correlated with the effective dust temperature, to a degree that it is only detected toward the coldest cores in the sample. However, we found two starless cores in our sample with similar column densities, but no $\mathrm{N}_{2} \mathrm{H}^{+}$detections (35 and 87), and these cores are significantly warmer than those that do have $\mathrm{N}_{2} \mathrm{H}^{+}$detections. Here, the column density may be due at least partly to chance projections and superpositions of otherwise unrelated material, even though in this case the column densities are higher than the minimum threshold for the detection of $\mathrm{N}_{2} \mathrm{H}^{+}$in cores found by Bergin et al. (2001) and reported as $A_{V}=4$ mag.

In summary, of common core tracers, $\mathrm{N}_{2} \mathrm{H}^{+}$is the only species to exclusively trace the coldest and densest cores, while the widely detected transitions of $\mathrm{HCN}, \mathrm{HCO}^{+}, \mathrm{HNC}$ and their isotopologs can be used to obtain a census of the total core population. The routine availability of Herschel effective dust temperatures for large areas of nearby star-forming regions is now providing an excellent starting point for targeted molecular-line studies of the coldest and densest cores.
Acknowledgements. We would like to thank Ali Ahmad Khostovan, who worked with us on this project as a summer student in the SAO REU Summer Intern Program in 2011, an anonymous referee for comments that helped to improve the paper, and Malcolm Walmsley for a careful reading of the manuscript and additional constructive suggestions for improvement. The Mopra Radio Telescope is part of the Australia Telescope National Facility, which is funded by the Commonwealth of Australia for operation as a National Facility managed by CSIRO. The University of New South Wales Digital Filter Bank used for the observations with the Mopra Telescope was provided with support from the Australian Research Council. Herschel is an ESA space observatory with science instruments provided by European-led Principal Investigator consortia and with important participation from NASA. This publication is supported by the Austrian Science Fund (FWF).

\section{References}

Alves, J., Lombardi, M., \& Lada, C. J. 2007, A\&A, 462, L17

Bergin, E. A., Ciardi, D. R., Lada, C. J., Alves, J., \& Lada, E. A. 2001, ApJ, 557, 209

Bergin, E. A., Alves, J., Huard, T., \& Lada, C. J. 2002, ApJ, 570, L101

Bisschop, S. E., Fraser, H. J., Öberg, K. I., van Dishoeck, E. F., \& Schlemmer, S. 2006, A\&A, 449, 1297

Burke, J. R., \& Hollenbach, D. J. 1983, ApJ, 265, 223

Caselli, P., Benson, P. J., Myers, P. C., \& Tafalla, M. 2002, ApJ, 572, 238

Forbrich, J., Lada, C. J., Muench, A. A., Alves, J., \& Lombardi, M. 2009, ApJ, 704, 292

Forbrich, J., Posselt, B., Covey, K. R., \& Lada, C. J. 2010, ApJ, 719, 691

Frau, P., Girart, J. M., Beltrán, M. T., et al. 2010, ApJ, 723, 1665

Frau, P., Girart, J. M., \& Beltrán, M. T. 2012a, A\&A, 537, L9

Frau, P., Girart, J. M., Beltrán, M. T., et al. 2012b, ApJ, 759, 3

Goldsmith, P. F. 2001, ApJ, 557, 736

Gonçalves, J., Galli, D., \& Walmsley, M. 2004, A\&A, 415, 617

Hacar, A., Tafalla, M., Kauffmann, J., \& Kovács, A. 2013, A\&A, 554, A55

Lada, C. J., Muench, A. A., Rathborne, J., Alves, J. F., \& Lombardi, M. 2008, ApJ, 672, 410

Ladd, N., Purcell, C., Wong, T., \& Robertson, S. 2005, PASA, 22, 62

Lombardi, M., Alves, J., \& Lada, C. J. 2006, A\&A, 454, 781

Lombardi, M., Bouy, H., Alves, J., \& Lada, C. J. 2014, A\&A, 566, A45

Marsh, K. A., Griffin, M. J., Palmeirim, P., et al. 2014, MNRAS, 439, 3683

Muench, A. A., Lada, C. J., Rathborne, J. M., Alves, J. F., \& Lombardi, M. 2007, ApJ, 671, 1820

Rathborne, J. M., Lada, C. J., Muench, A. A., Alves, J. F., \& Lombardi, M. 2008, ApJS, 174, 396

Rathborne, J. M., Lada, C. J., Muench, A. A., et al. 2009, ApJ, 699, 742

Román-Zúñiga, C. G., Alves, J. F., Lada, C. J., \& Lombardi, M. 2010, ApJ, 725, 2232

Tafalla, M., Santiago-García, J., Myers, P. C., et al. 2006, A\&A, 455, 577

Ward-Thompson, D., André, P., \& Kirk, J. M. 2002, MNRAS, 329, 257 
Table 1. Integrated line intensities and core temperatures.

\begin{tabular}{|c|c|c|c|c|c|c|c|c|c|c|}
\hline Core $^{a}$ & $\begin{array}{r}\ell \\
\left({ }^{\circ}\right)\end{array}$ & $\begin{array}{r}b \\
\left({ }^{\circ}\right)\end{array}$ & $\begin{array}{r}A_{V} \\
(\mathrm{mag})\end{array}$ & $\mathrm{N}_{2} \mathrm{H}^{+}$ & $\mathrm{HCN}$ & $\begin{array}{l}\mathrm{H}^{13} \mathrm{CN} \\
\quad(\mathrm{K} \mathrm{kn}\end{array}$ & $\begin{array}{l}\mathrm{HNC} \\
\left(\mathrm{s}^{-1}\right)\end{array}$ & $\mathrm{HCO}^{+}$ & $\mathrm{H}^{13} \mathrm{CO}^{+}$ & $\begin{array}{r}T_{\text {eff }} \text { (dust) } \\
(\mathrm{K})\end{array}$ \\
\hline $3^{b}$ & -3.03 & 7.28 & 22.0 & $0.18(3)$ & $0.17(3)$ & & $0.17(2)$ & $0.36(2)$ & $0.16(1)$ & $14.55(13)$ \\
\hline 4 & -2.99 & 7.01 & 12.8 & & $0.18(3)$ & & $0.25(2)$ & $0.63(2)$ & $0.09(1)$ & $15.31(12)$ \\
\hline $5^{b}$ & -2.97 & 6.85 & 11.9 & & $0.17(5)$ & & & $0.22(5)$ & & $15.48(14)$ \\
\hline 7 & -2.95 & 7.25 & 9.6 & & $0.20(9)$ & & $0.30(6)$ & $0.61(6)$ & & $15.80(14)$ \\
\hline $9^{b}$ & -2.93 & 7.12 & 67.3 & $2.25(8)$ & $0.65(8)$ & $0.35(8)$ & $1.16(8)$ & $1.40(6)$ & $0.79(6)$ & $13.50(21)$ \\
\hline 10 & -2.83 & 7.35 & $4.8^{c}$ & & $0.09(3)$ & & $0.20(3)$ & $0.23(2)$ & & $17.51(21)$ \\
\hline $11^{b}$ & -2.71 & 6.96 & 11.0 & & $0.17(4)$ & & $0.20(3)$ & $0.46(3)$ & $0.03(1)$ & $14.74(11)$ \\
\hline 12 & -2.68 & 6.78 & $6.9^{c}$ & & $0.18(5)$ & & & $0.30(7)$ & & $16.55(19)$ \\
\hline $16^{b}$ & -2.54 & 6.35 & 11.7 & & $0.15(4)$ & & $0.39(4)$ & $0.25(5)$ & & $15.87(12)$ \\
\hline 17 & -2.42 & 6.51 & 7.4 & & & & $0.12(4)$ & $0.29(3)$ & & $17.44(13)$ \\
\hline 18 & -2.38 & 6.22 & 9.0 & & & & & & & $16.90(13)$ \\
\hline 23 & -1.84 & 6.30 & 9.0 & & & & & $0.06(2)$ & & $16.85(13)$ \\
\hline 28 & -1.46 & 5.47 & 6.4 & & & & $0.12(2)$ & $0.15(3)$ & & $17.77(26)$ \\
\hline $29^{b}$ & -1.43 & 5.90 & 13.5 & & $0.15(5)$ & & $0.11(4)$ & & & $15.74(13)$ \\
\hline 30 & -1.41 & 5.75 & 10.5 & & $0.06(4)$ & & $0.18(3)$ & $0.17(3)$ & & $16.53(13)$ \\
\hline 33 & -1.28 & 6.04 & 12.7 & & & & & $0.45(6)$ & & $15.54(11)$ \\
\hline $34^{b}$ & -1.21 & 5.64 & 20.3 & $0.27(3)$ & $0.06(2)$ & & $0.46(2)$ & $0.18(2)$ & $0.13(1)$ & $14.26(07)$ \\
\hline 35 & -1.19 & 5.26 & 20.6 & & $0.16(3)$ & & $0.16(2)$ & $0.27(3)$ & & $15.51(08)$ \\
\hline 38 & -0.52 & 5.24 & 6.5 & & & & & & & $17.45(17)$ \\
\hline $39^{b}$ & -0.50 & 4.44 & 10.0 & & & & $0.08(4)$ & $0.21(5)$ & & $16.44(15)$ \\
\hline $40^{b}$ & -0.49 & 4.85 & 10.1 & & $0.11(4)$ & & & $0.20(2)$ & & $16.59(13)$ \\
\hline 43 & -0.31 & 4.58 & 7.9 & & & & & $0.29(5)$ & & $17.05(16)$ \\
\hline $46^{b}$ & 0.07 & 4.62 & 12.9 & & & & & $0.22(7)$ & & $16.18(16)$ \\
\hline 47 & 0.08 & 3.86 & 8.8 & & & & & & & $18.32(19)$ \\
\hline 51 & 0.23 & 4.55 & 10.4 & & & & & $0.13(3)$ & & $16.71(13)$ \\
\hline 52 & 0.31 & 3.87 & 9.8 & & & & & $0.13(3)$ & & $17.57(14)$ \\
\hline $53^{b}$ & 0.37 & 3.97 & 12.1 & & & & & $0.20(2)$ & & $16.64(15)$ \\
\hline 55 & 0.53 & 4.78 & 9.1 & & $0.09(4)$ & & $0.09(3)$ & $0.23(4)$ & & $16.73(15)$ \\
\hline 56 & 0.59 & 4.48 & 8.0 & & & & & $0.14(4)$ & & $17.60(22)$ \\
\hline $60^{b}$ & 0.73 & 3.87 & 13.0 & & & & & & & $16.48(11)$ \\
\hline 68 & 1.20 & 3.57 & 9.4 & & $0.07(3)$ & & & $0.11(2)$ & & $18.33(17)$ \\
\hline $71^{b}$ & 1.31 & 3.76 & 49.2 & $0.68(3)$ & $0.30(2)$ & $0.04(2)$ & $1.01(2)$ & $0.25(3)$ & $0.24(2)$ & $13.18(06)$ \\
\hline 72 & 1.33 & 3.93 & 11.6 & & & & & & & $17.03(11)$ \\
\hline 74 & 1.38 & 4.40 & 13.5 & & $0.14(3)$ & & $0.27(3)$ & $0.60(2)$ & $0.17(2)$ & $16.41(13)$ \\
\hline 76 & 1.41 & 3.71 & 14.4 & & $0.16(3)$ & & $0.20(2)$ & $0.30(2)$ & & $16.23(13)$ \\
\hline 81 & 1.47 & 4.10 & 15.3 & & $0.18(5)$ & & $0.27(4)$ & $0.16(4)$ & & $17.15(10)$ \\
\hline 82 & 1.48 & 3.79 & 15.1 & & & & & $0.26(3)$ & & $16.91(11)$ \\
\hline 84 & 1.51 & 6.41 & 7.7 & & $0.18(9)$ & & & $0.24(3)$ & & $17.64(40)$ \\
\hline 86 & 1.52 & 7.08 & 17.5 & $0.16(3)$ & $0.20(6)$ & & $0.51(5)$ & $0.62(2)$ & $0.14(2)$ & $14.32(19)$ \\
\hline $87^{b}$ & 1.52 & 3.92 & 23.0 & & $0.11(5)$ & & $0.17(3)$ & $0.37(6)$ & & $15.86(08)$ \\
\hline 90 & 1.58 & 6.43 & 9.4 & & & & & $0.34(4)$ & & $16.95(20)$ \\
\hline 91 & 1.58 & 6.49 & 11.8 & & & & & $0.46(4)$ & & $16.78(19)$ \\
\hline $96^{b}$ & 1.71 & 3.65 & 42.9 & $0.82(5)$ & $0.45(4)$ & $0.10(4)$ & $1.07(4)$ & $0.75(2)$ & $0.27(3)$ & $13.76(07)$ \\
\hline 99 & 1.77 & 6.93 & 10.1 & & $0.12(6)$ & & & $0.16(4)$ & & $16.56(16)$ \\
\hline 100 & 1.77 & 6.98 & 9.3 & & $0.15(4)$ & & & $0.18(2)$ & & $16.67(16)$ \\
\hline 101 & 1.80 & 3.88 & 10.3 & & & & & & & $18.38(16)$ \\
\hline 102 & 1.80 & 7.15 & 6.1 & & & & & & & $18.29(28)$ \\
\hline 103 & 1.85 & 3.76 & 11.8 & & $0.18(4)$ & & & $0.24(2)$ & & $17.37(13)$ \\
\hline 110 & 2.00 & 3.63 & 12.8 & & & & & $0.24(5)$ & & $17.99(14)$ \\
\hline 115 & 2.13 & 3.55 & 11.6 & & & & & & & $18.30(15)$ \\
\hline 117 & 2.22 & 3.35 & 11.1 & & $0.19(5)$ & & & $0.14(3)$ & & $18.39(12)$ \\
\hline 118 & 2.22 & 3.41 & 10.2 & & $0.19(5)$ & & & $0.20(3)$ & & $17.96(15)$ \\
\hline
\end{tabular}

Notes. $1 \sigma$ errors in the last digit(s) shown in brackets (see text for a discussion of the temperature errors). Empty fields denote non-detections. ${ }^{(a)}$ Core numbers are from Rathborne et al. (2009). ${ }^{(b)}$ Cores also discussed by Frau et al. (2010, 2012a,b), but using core numbers from Rathborne et al. (2008) instead of Rathborne et al. (2009). The corresponding numbers are as follows (Frau numbers in brackets): 3(6), 5(8), 9(12), 11(14), 16(20), 29(33), 34(40), 39(47), 40(48), 46(56), 53(65), 60(74), 71(87), 87(102), and 96(109). ${ }^{(c)}$ Extinction data from Lombardi et al. (2006) instead of Román-Zúñiga et al. (2010). 